\title{
Citizen science with smart gas sensors: air quality assessment and environmental studies by students
}

\author{
Sebastian Höfner ${ }^{1}$, Benjamin Brück ${ }^{1,2}$, Michael Hirth ${ }^{3}$, Jochen Kuhn ${ }^{3}$, Andreas Schütze ${ }^{1}$ \\ ${ }^{1}$ Laboratory for Measurement Technology, Saarland University, Saarbrücken, Germany \\ 2 Schülerforschungszentrum Saarlouis, Saarlouis, Germany \\ ${ }^{3}$ Physics Education Research Group, Department of Physics, University of Kaiserslautern, Germany \\ schuetze@Imt.uni-saarland.de
}

\begin{abstract}
Harmful gases often are odorless as well as invisible and therefore only recognizable with gas sensors. This leads to limited awareness of possible hazards in the general population. In a novel project, we aim to introduce high school students to modern micro gas sensors allowing them to use smart sensor systems coupled with a smartphone app for air quality assessment and environmental studies. In three modules students learn how a metal oxide gas sensor works, how to calibrate a sensor and to evaluate data from environmental measurements with mobile devices. The ultimate aim is to increase the students environmental awareness for air pollution in general and especially concerning the effects of our own behavior, with this citizen science inspired approach.
\end{abstract}

Key words: gas sensors, smartphones, mobile assisted seamless learning, environmental studies

\section{Introduction}

In 2012 over a half million deaths in Europe were attributed to air pollution [1]. Although awareness of human responsibility is increasing continuously, it is still somewhat diffuse and abstract for many people, especially for high school students. A key problem is that there is no direct perception of air quality or hazardous gases and, thus, no immediate feedback of the ecological damage of human activities. Regarding our personal impact on the environment teaching of appropriate competences and systemic connections as well as promoting awareness and sustainable behavior is a key prerequisite for achieving a sustainable society.

\section{Aim of the project}

The main goal of the project is to increase the environmental awareness of high school students (ages 14 to 18 ) by combining modern smartphones and tablets (cool gadgets they use every day) with smart gas sensors developed for IoT applications. The central approach here is to make use of the diverse sensor spectrum in modern mobile devices to attract young people's interest, but at the same time to perform measurements and support the documentation of results.

Students are introduced to integrated micro gas sensors (i.e. BME680 by Bosch SensorTec or the Multi-Pixel gas sensor SGP30 by Sensirion) as well as the need for and approach to their calibration. Finally, students are motivated to make use of mobile environmental measurement technology to develop their own research questions after the initial training and to examine them in environmental studies.

\section{Didactic concept}

The didactic concept combines three aspects:

- Authentic Learning: The measurement of air pollutants represents an authentic measurement task due to the realistic conditions and use of common devices, i.e. smartphones.

- Education for Sustainable Development: Respect for nature and our responsibility for its preservation is communicated.

- Mobile Assisted Seamless Learning: The mobility and ubiquity of smartphones and tablets make it possible to experience learning as a process that is not limited to the classroom. This means that learning can take place anytime, anywhere, formally and informally, individually and cooperatively [2].

\section{Experiments}

The project is divided into three main modules.

\section{I: Functionality of semiconductor gas sensors}

In the first module, the students are introduced to the function principle of a metal oxide gas 
sensor. The gas-specific temperature dependence of the sensor reaction is explained and the principle of Temperature Cycled Operation (TCO) is introduced to increase the sensitivity, selectivity and stability and to allow the design of application specific smart sensor systems [3].

For this module a simple experimental platform to understand the fundamental functionality of a semiconductor gas sensor was developed. The students receive a simple circuit board with an conventional analog gas sensor. By adjusting the heater voltage and simultaneously measuring the sensor resistance with an ohmmeter students are able to observe the resistance change in the presence of a gas.

The limit of this simple setup is demonstrated by comparing two different substances with the same outcome. This leads to the next module in which a temperature cycle is used to discriminate different gases and gas concentrations.

\section{II: Indoor air quality}

The second module is focused on Indoor Air Quality (IAQ). Students will measure different gases inside (school) rooms which are responsible for health effects, e.g. the "sick building syndrome" [4]. Relevant target gases are:

- Inorganic compounds, e.g. $\mathrm{CO}, \mathrm{NO}_{x}$, ozone

- VOCs, e.g. tVOC, formaldehyde, benzene

- $\mathrm{CO}_{2}$ (using NDIR sensor modules)

The data are recorded and evaluated using smartphone apps. In this module the students will first calibrate a sensor with different Ethanol concentrations. The calibration is supported by the microcontroller with a program implementting a partial least squares regression (PLSR) model.

\section{III: Environmental studies}

After carrying out the introductory experiments in student laboratories and school lessons, students are encouraged to work on selected environmental issues and to develop their own questions which can be investigated by appropriate mobile measurement methods.

\section{Possible topics are:}

- Indoor air quality in different class rooms

- Exhaust emissions of old and new cars

- Air quality in the city and the countryside

Some of the possible following experiments are predefined, but mainly the students are encouraged to develop their own research questions.

\section{Implementation}

All modules will be accompanied by HTML tutorials. This increases the students motivation and allows every student to perform the experi- ments at his or her own speed, which leads to a better learning success.

The smart sensor, consisting of an integrated gas sensor coupled with a microcontroller, will be programmed and calibrated for the desired task. All measurement data are recorded and sent to an online server for storage and further processing. Apps, written for mobile devices, will communicate with the server to download and display the recorded data.

An example of an app-based visualization of sensor data is shown in figure 1 .

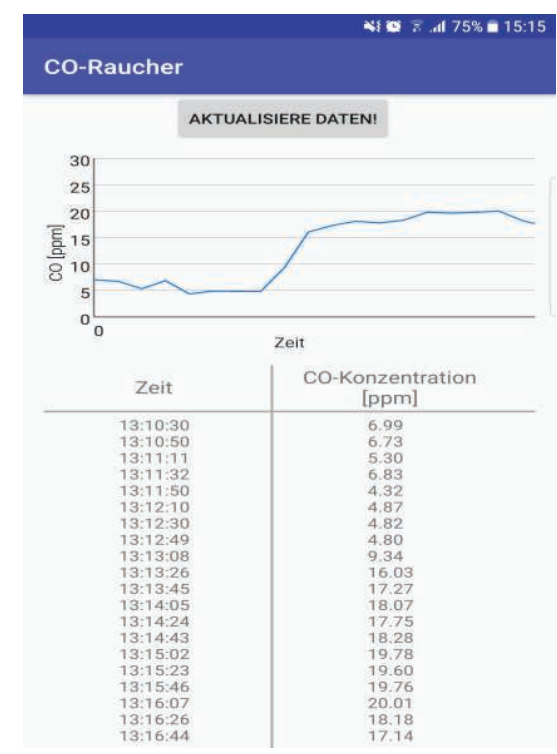

Figure 1: Example for an app based visualization of sensor data over time. Here, the CO concentration in exhaled breath is determined to identify smokers.

\section{Outlook}

Module three will focus on citizen science. Data sets will be publicly available to increase the interest in environmental issues as well as the motivation of the student researchers. The goal will be the creation of a user-friendly web interface where the data is presented.

\section{References}

[1] Air Quality in Europe - 2015 report, European Environmental Agency, doi:10.2800/62459

[2] Wong, L. H. \& Looi, C. K. (2011). What seams do we remove in mobile assisted seamless learning? A critical review of the literature. Computers and Education, 57 (4), 2364-2381.

[3] Reimann, P. \& Schütze, A. (2014): Sensor Arrays, Virtual Multisensors, Data Fusion, and Gas Sensor Data Evaluation. In: C.-D. Kohl, T. Wagner (eds.): Gas Sensing Fundamentals, Springer Series on Chemical Sensors and Biosensors, Volume 15.

[4] M. Hodgson. Indoor environmental exposures and symptoms. In: Environ Health Perspect. 2002 Aug;110 Suppl 4, S. 663-667. Review. PMID 12194903 